\title{
Management of paraproteinaemia
}

\author{
Lucy Cook, Donald H C Macdonald
}

Postgrad Med J 2007;83:217-223. doi: 10.1136/pgmj.2006.054627

A paraprotein is a monoclonal immunoglobulin or light chain present in the blood or urine; it is produced by a clonal population of mature B cells, most commonly plasma cells. In individuals aged $>50$ years the incidence of a paraprotein is $3.2 \%$. Plasma cell disorders can be considered as a spectrum of conditions ranging from monoclonal gammopathy of undetermined significance (MGUS), through asymptomatic, to symptomatic myeloma. MGUS is defined by a low level of paraprotein $<30 \mathrm{~g} / \mathrm{l}$, bone marrow plasma cells $<10 \%$ and the absence of myeloma related organ or tissue damage (predominantly renal, skeletal or bone marrow impairment.) MGUS requires no therapy and the overall risk of progression to myeloma is $1 \%$ per year. Myeloma remains incurable with a median survival of 3-4 years; autologous stem cell transplant can prolong survival, if appropriate. Thalidomide in combination with dexamethasone has an emerging role in the treatment of myeloma.

See end of article for authors' affiliations

Correspondence to:

Correspondence to:
Dr Donald Macdonald, Room $1 \mathrm{~L} 05$, Charing Cross Hospital, Fulham Palace

Road, London W6 8RF, UK; d.h.macdonald@imperial. ac.uk

Received 24 October 2006 Accepted 5 January 2007
A paraprotein is a monoclonal immunoglobulin or immunoglobulin light chain (Bence Jones protein) present in the blood or urine and arising from clonal proliferation of mature Bcells, most commonly plasma cells or B-lymphocytes. Alternative terms include monoclonal protein or M-band. Paraproteins are characterised by homogenous electrophoretic migration and the expression of a single light chain type, either $\kappa$ or $\lambda$. There are several situations in which a paraprotein may be identified and these scenarios have differing clinical significance. At one extreme there may be an overtly malignant clonal proliferation of plasma cells resulting in multiple myeloma, or solitary plasmacytoma (skeletal or extramedullary.) At the other extreme there may be a low level paraprotein ultimately classified as monoclonal gammopathy of undetermined significance (MGUS) which may be of little clinical relevance. The aim of this article is to aid the physician in developing a strategy which can identify the likely clinical condition underlying a paraprotein, deal with the important clinical scenario of distinguishing between MGUS and multiple myeloma, and provide a brief overview of important clinical aspects of multiple myeloma.

\section{WHAT IS THE INCIDENCE OF PARAPROTEINAEMIA?}

Recently published population based studies from Minnesota have clarified the incidence of paraproteinaemia in the general population. In individuals aged $>50$ years the overall incidence of a paraprotein is $3.2 \%$; this varies with age (age 50 $59,1.7 \%$; age $>70,5.3 \%$ ) and sex (men: women, 4.0\%: $2.7 \%$. $)^{1}$ There is also an ethnic variation with previous reports noting the age adjusted prevalence of MGUS being threefold higher in African Americans than the white population. ${ }^{2}$ Paraproteins are therefore a common laboratory finding in an elderly population.

\section{DISORDERS ASSOCIATED WITH A PARAPROTEIN}

A wide range of mature $\mathrm{B}$ cell disorders may be associated with a circulating paraprotein (table 1). In a review of more than 1000 cases of M-protein seen at the Mayo Clinic the underlying diagnosis was MGUS (51\%), multiple myeloma (18\%), AL amyloidosis $(11 \%)$, lymphoproliferative disorder $(4 \%)$, and other disorders such as smouldering myeloma or solitary plasmacytoma $(16 \%){ }^{3}$ The lymphoproliferative disorders chronic lymphocytic leukaemia (CLL) and lymphoplasmacytic lymphoma/Waldenstrom macroglobulinaemia (LPL/ WM) generally have associated clinical and laboratory features which greatly facilitate diagnosis. CLL can infrequently be associated with a paraprotein. In practice CLL is usually diagnosed as a result of investigating a persisting monoclonal peripheral blood lymphocytosis and generalised lymphadenopathy. Immunophenotyping of the circulating B lymphocytes in CLL reveals a characteristic pattern of surface antigen expression. This profile has been used to formulate a CLL diagnostic score based on the expression of CD23, CD5, FMC7, CD79b/CD22, and SIg. Immunophenotyping by flow cytometry of a persisting lymphocytosis is now routinely performed to establish the diagnosis of CLL. ${ }^{4}$ The other nonHodgkin's lymphoma commonly associated with a paraprotein is LPL/WM, a rare disorder (15\% of all lymphomas) which presents at a median age of 63. There is lymphomatous infiltration of nodes, bone marrow, or spleen and the majority of patients have a circulating $\operatorname{IgM}$ paraprotein $>30 \mathrm{gm} / \mathrm{l}$. Of particular note, IgM paraproteins may have autoantibody or cryoglobulin activity resulting in peripheral neuropathy $(10 \%$ of patients) or cryoglobulinaemia. Additionally high

\footnotetext{
Abbreviations: $\mathrm{CC}$, conventional chemotherapy; $\mathrm{CL}$, chronic lymphocytic leukaemia; C-VAMP, cyclophosphamide, vincristine, adriamycin and prednisolone; HDT, high dose therapy; IPI, international prognostic index; LPL/WM, lymphoplasmacytic lymphoma/ Waldenstrom macroglobulinaemia; MGUS, monoclonal gammopathy of undetermined significance; MP, melphalan and prednisolone; NHL, non-Hodgkin's lymphoma; PBSC, peripheral blood stem cell collection; ROTI, related organ or tissue impairment; VAD, vincristine and doxorubicin
} 
levels of circulating IgM pentamers cause hyperviscosity (10$30 \%$ of patients) with reduced visual acuity and increased risk of cerebrovascular accidents. ${ }^{5}$ Therefore the finding of a high concentration IgM paraprotein should raise the suspicion of LPL and may require urgent specialist investigation and treatment to prevent the complications of hyperviscosity. The diagnosis and management of CLL and LPL/WM is beyond the scope of this article and is dealt with fully in the British Society for Haematology Guidelines (www.bcshguidelines.com).

By definition all paraproteins are the product of a B cell clone which may be large, small or possibly undetectable using available techniques. The disorders described above are examples of $\mathrm{B}$ cell disorders where the clinical features are defined either by the systemic effects of expansion of the malignant clone or which may be entirely asymptomatic and meet the diagnostic criteria of MGUS. Alternatively, in some B cell conditions, while the clone may be undetectable the clinical features are dominated by the biological effects of the paraprotein, so called "dangerous small B-cell clones" ${ }^{6}{ }^{6}$ There

\section{Box 1 Disorders associated with a paraprotein}

Malignant B-cell disorders

- Multiple myeloma $(\lg G, \lg A, \lg D, \lg E$, or $\kappa, \lambda$ free light chains)

Symptomatic myeloma

Asymptomatic myeloma

Plasma cell leukaemia

Non-secretory myeloma

- POEMS

Polyneuropathy, organomegaly, endocrinopathy, Mprotein, skin changes

- Plasmacytoma

Solitary plasmacytoma of bone

Extramedullary

- Lymphoproliferative disorders

Chronic lymphocytic leukaemia

Non-Hodgkin lymphomas including lymphoplasmacytic lymphoma

(Waldenstrom macroglobulinaemia)

- Heavy chain disease

$\gamma, \alpha, \mu$

- Amyloidosis (AL)

MGUS

- Paraprotein detected with no evidence of other B cell disorder

Non-malignant systemic disease

- Autoimmune disease Rheumatoid arthritis

Scleroderma

Hashimoto thyroiditis

- Cutaneous disease Pyoderma gangrenosum Necrobiotic xanthogranulomatosis

- Liver disease Hepatitis/cirrhosis

- Infectious disease Mycobacterium tuberculosis Bacterial endocarditis

MGUS, monoclonal gammopathy of undetermined significance are two pathogenetic mechanisms for this type of disorder. Either the monoclonal protein may aggregate and deposit systemically, causing disorders such as light-chain amyloidosis, crystal storing histiocytosis or cryoglobulinaemia type I. Alternatively the monoclonal protein may have antibody activity towards an autogenous antigen causing disorders such as polyneuropathy, monoclonal cold agglutinins and cryglobulinaemia type II.

The final group of disorders are paraproteins found in association with a non-malignant systemic disorder. In these circumstances neither the small B cell clone nor the monoclonal protein causes the predominant clinical features, but the case cannot be labelled as MGUS because of the presence of a coexisting systemic disorder. These paraproteins are often small and may occasionally be transient. Examples include paraproteins associated with autoimmune, liver and skin diseases as listed in box 1 .

\section{WHEN SHOULD ONE TEST FOR THE PRESENCE OF A PARAPROTEIN?}

Multiple myeloma is the most common clinically important disorder associated with a paraprotein, and may have a wide range of clinical presentations. It is therefore prudent that a patient should be screened for the presence of an M-protein when presenting with any of the clinical findings listed in box 2 .

It is an important practice point to emphasise that if considering myeloma as a diagnosis then both the urine and blood must be screened for a paraprotein, because in 15\% of myeloma cases a monoclonal immunoglobulin light chain may be the only M-protein present. Such cases would be missed if serum protein electrophoresis were the only investigation performed. The measurement of serum-free light chains introduced relatively recently into clinical practice allows the quantification of free $\kappa$ and $\lambda$ light chains secreted by plasma cells. An abnormal $\kappa: \lambda$ ratio suggests an excess of one light chain type and, although the mechanism is poorly understood, is used as a surrogate marker for clonal expansion. The use of such a serum assay will abrogate the need to test both blood and urine for diagnosis.

\section{HOW SHOULD A PATIENT WITH PARAPROTEINAEMIA BE INVESTIGATED FURTHER?}

As noted above, the underlying disorders associated with a paraprotein can broadly be divided into non-Hodgkin lymphoma subtypes and plasma cell disorders. The lymphoproliferative disorders CLL and LPL/WM do not generally present

Box 2 Clinical indications for screening for $M$ protein

- Malaise and fatigue

- Bone disease (persistent back pain, osteopenia or lytic lesions)

- Impaired renal function

- Normochromic normocytic anaemia \pm pancyłopenia

- Hypercalcaemia

- Recurrent bacterial infections

- Hyperviscosity

- Nephrotic syndrome, cardiac failure, malabsorption

- Peripheral neuropathies, carpal tunnel syndrome

- Incidental persistent elevated erythrocyte sedimentation rate 
Table 1 Diagnostic criteria for monoclonal gammopathy of undetermined significance (MGUS), asymptomatic myeloma and symptomatic myeloma (based upon BCSH guidelines 2005)

\begin{tabular}{|c|c|c|c|}
\hline & MGUS & $\begin{array}{l}\text { Asymptomatic } \\
\text { myeloma }\end{array}$ & $\begin{array}{l}\text { Symptomatic } \\
\text { myeloma }\end{array}$ \\
\hline $\begin{array}{l}\text { Paraprotein } \\
\text { Bone marrow clonal } \\
\text { plasma cells }\end{array}$ & $\begin{array}{l}<30 \mathrm{~g} / \mathrm{l} \\
<10 \%\end{array}$ & $\begin{array}{l}>30 \mathrm{~g} / \mathrm{l} \\
>10 \%\end{array}$ & $\begin{array}{l}\text { Variable* } \\
>10 \%\end{array}$ \\
\hline $\begin{array}{l}\text { Bence Jones protein } \\
\text { Immune paresis } \\
\text { ROTI: }\end{array}$ & $\begin{array}{l}\text { Possible } \\
\text { Possible }\end{array}$ & $\begin{array}{l}\text { Possible } \\
\text { Probable }\end{array}$ & $\begin{array}{l}50 \% \text { of cases } \\
\text { Probable }\end{array}$ \\
\hline Lytic Lesions & Absent & Absent & Present \\
\hline Anaemia & Absent & Absent & $66 \%$ of cases \\
\hline Renal dysfunction & Absent & Absent & $30 \%$ of cases \\
\hline Hypercalcaemia & Absent & Absent & $30 \%$ of cases \\
\hline Symptoms & Absent & Absent & Frequent \\
\hline
\end{tabular}

*Diagnosis made in conjunction with other features.

diagnostic difficulties and are generally referred to a haematooncologist. In clinical practice the most frequent scenario is the assessment of a paraprotein found in association with a plasma cell disorder. The significance of such a finding can range from a medical emergency to a coincidental finding of little clinical relevance. All patients with an $\mathrm{M}$-protein should be referred for specialist review. The goal of such review will be to establish the precise diagnosis this generally entails distinguishing between MGUS, asymptomatic, or symptomatic myeloma, with consideration of other linked diagnoses such as solitary plasmacytoma and/or AL amyloidosis. The degree of urgency for such referrals varies: clearly those with associated renal failure, hypercalcaemia, or cord compression are medical emergencies requiring immediate specialist treatment; those patients with suspected myeloma should be referred for urgent specialist advice; and those in whom a paraprotein is detected routinely should be referred for non-urgent advice as they will require long term follow-up. It is also prudent to remember that in an elderly population a paraprotein may well be a clinically insignificant MGUS with a co-existent second pathology. Consequently, if anaemia is detected then haematinics should be checked, and if iron deficiency is present then gastroenterological investigation is required to exclude blood loss. Similarly, if hypercalcaemia is present then primary hyperparathyroidism should be excluded.

\section{HOW IS MGUS DISTINGUISHED FROM MYELOMA?}

In patients with a plasma cell disorder and a circulating paraprotein, current practice is to subdivide these patients into three groups: MGUS, asymptomatic myeloma (previously termed smouldering, indolent or equivocal myeloma), and symptomatic myeloma. The distinction is important as it determines prognosis, and the need for treatment. Three variables define these groups: (1) M-protein level, (2) bone marrow plasma cell percentage, and (3) the presence or absence of myeloma related organ or tissue impairment (ROTI). The relevant investigations are therefore to estimate $\mathrm{M}$-protein levels: serum protein electrophoresis, immunofixation and paraprotein quantification plus $24 \mathrm{~h}$ urinary light chain quantification. Bone marrow aspirate and trephine biopsy are also required to determine the extent of bone marrow infiltration. It should, however, be emphasised that while plasma cell percentage is part of the diagnostic criteria for MGUS, to avoid inappropriate over investigation bone marrow biopsy is not required in all cases where a circulating paraprotein is detected. This is detailed below in the section managing MGUS. These basic investigations are supplemented with tests to establish the presence or absence of ROTI. The diagnostic criteria for MGUS and myeloma are set out in table 1 . The clinicopathological features of myeloma ROTI are set out in table 2. The necessary investigations include full blood count, assessment of renal function, corrected calcium, and skeletal imaging, most commonly a skeletal survey. In certain circumstances, as detailed below, further imaging may be required. Two important practice points should be emphasised; in a patient with a circulating paraprotein, low levels of Bence Jones protein and an immune paresis of the polyclonal immunoglobulins are compatible with the diagnosis of MGUS. Although the term symptomatic myeloma is used, in any patient with laboratory criteria for myeloma the presence of myelomarelated skeletal damage, though it may not be causing symptoms, is evidence of ROTI and would automatically classify the patient as having symptomatic myeloma. ${ }^{7}$

\section{WHAT IS THE PROGNOSIS AND HOW DO WE MANAGE MGUS?}

MGUS is defined as an M-protein in the serum $<30 \mathrm{~g} / \mathrm{l}$, clonal plasma cells in the bone marrow $<10 \%$, and absence of myeloma ROTI. Clearly it is inappropriate to perform a skeletal survey and bone marrow investigation in all patients in whom a low level paraprotein is detected. Clinical judgement is required and current UK practice in the investigation of MGUS is to reserve these investigations for younger patients and those in whom the paraprotein is $>20 \mathrm{~g} / \mathrm{l}$. Characteristically, individuals with MGUS have no symptoms or signs related to the monoclonal population and MGUS does not require treatment; they do, however, require indefinite follow up, the details of which are set out below. Occasional patients have monoclonal antibodies that are directed against coagulation proteins resulting in Von Willebrand disease; against insulin, resulting in hypoglycaemia; and against peripheral nerve myelin resulting in peripheral neuropathy. ${ }^{8}$

Overall the risk of progression of MGUS to myeloma or related disorder is $1 \%$ per year. Although the prevalence of MGUS increases with advancing age, after adjustment for the level of the M-protein, the annual risk of progression to myeloma or a related cancer is not affected by age or the duration of MGUS. Younger patients are more likely to have progression to cancer during their lifetime because they are at risk for longer. In contrast to age and duration, other factors, notably the level of M-protein, do predict the risk of progression. The percentage risk of progression in 10 years roughly equates to the level of the M-protein-for example, $20 \mathrm{~g} / \mathrm{l}$ is associated with $20 \%$ risk at 10 years of malignancy. However, in reality, when competing causes of death are taken into account, the true life-time probability of progression is substantially lower.

Given the uncertainty of disease progression with MGUS attempts have been made to identify those patients at highest risk. The parameters which are useful for predicting the likelihood of progression are: the size of the M-protein, ${ }^{10}$ the type of the M-protein with IgA and IgM paraproteins having a higher risk compared to IgG paraproteins, ${ }^{11}$ the percentage of bone marrow plasma cells, ${ }^{12}$ and an abnormal serum-free light chain ratio. A large population based study suggested that the risk of progression to myeloma or related malignancy with an abnormal ratio at diagnosis was $17 \%$ at 10 years as compared to $5 \%$ at 10 years in those patients with a normal ratio. ${ }^{313} 14$

On the basis of these findings a risk stratification system set out in table 3 has recently been proposed for MGUS. High risk patients had a risk of progression of $58 \%$ at 20 years. The low risk group, which constitutes $40 \%$ of the cohort, carries a risk of only $5 \%$ at 20 years. In practice, when competing causes of death are taken into account in this predominately elderly population, the actual risk of progression is lower. On the basis 
Table 2 Myeloma-related organ or tissue impairment (ROTI). Based upon BCSH guidelines 2005

\begin{tabular}{|c|c|}
\hline $\begin{array}{l}\text { Increased } \\
\text { calcium levels }\end{array}$ & $\begin{array}{l}\text { Corrected serum calcium }>0.25 \mathrm{mmol} / \mathrm{l} \text { above the upper } \\
\text { limit of normal or }>2.75 \mathrm{mmol} / \mathrm{l}\end{array}$ \\
\hline $\begin{array}{l}\text { Renal } \\
\text { insufficiency }\end{array}$ & Attributable to myeloma \\
\hline Anaemia & $\begin{array}{l}\text { Haemoglobin } 2 \mathrm{~g} / \mathrm{dl} \text { below the lower limit of normal or } \\
\text { haemoglobin }<10 \mathrm{~g} / \mathrm{dl}\end{array}$ \\
\hline Bone lesions & $\begin{array}{l}\text { Lytic lesions or osteoporosis with compression fractures } \\
\text { (MRI or CT may clarify) }\end{array}$ \\
\hline Other & $\begin{array}{l}\text { Symptomatic hyperviscosity, amyloidosis, recurrent } \\
\text { bacterial infections (>2 episodes in } 12 \text { months) }\end{array}$ \\
\hline
\end{tabular}

of these findings current recommendations are that low risk patients are seen initially at 6 months and then once every 2 years if asymptomatic. At each follow-up visit patients are assessed by means of history and examination, full blood count, serum calcium, renal function and paraprotein quantification. There are currently phase I and II clinical trials of chemoprophylaxis underway for those with high risk MGUS. It must be emphasised that outside the setting of a clinical trial, no specific therapy is required for MGUS. ${ }^{3}$

\section{HOW DO WE INVESTIGATE AND MANAGE ASYMPTOMATIC AND SYMPTOMATIC MYELOMA?}

The initial investigations are as described previously: M-protein quantification, bone marrow biopsy, skeletal survey and investigations to identify the presence of myeloma ROTI. Additional imaging studies are often required. Magnetic resonance imaging (MRI) is an essential investigation for patients presenting with signs of spinal cord compression and is useful to assess the extent of soft tissue disease. Computed tomography (CT) scanning can be useful to clarify the significance of equivocal abnormalities on a plain radiograph, and is essential in planning radiotherapy. In addition the prognostic factors albumin and $\beta_{2}$ microglobulin are measured.

Asymptomatic myeloma accounts for approximately 15\% of all cases of newly diagnosed myeloma. Most patients will progress eventually to symptomatic disease-the median time to progression from asymptomatic to symptomatic myeloma is 12-32 months. All patients with myeloma are felt to evolve from MGUS/asymptomatic myeloma, although in many these pre-malignant stages are unrecognised clinically because of their asymptomatic nature. A known prior history of MGUS or asymptomatic myeloma has no impact on the prognosis of myeloma. No specific treatment is recommended for asymptomatic myeloma, but patients require follow-up at least once every 4 months. Patients should be educated about symptoms such as new or progressive bone pain, and persistent or recurrent infection, which might suggest disease progression.
Table 4 International prognostic index

\begin{tabular}{lll}
\hline Stage & Criteria & $\begin{array}{l}\text { Median survival } \\
\text { (months) }\end{array}$ \\
\hline I & $\beta_{2}$ microglobulin $<3.5 \mathrm{mg} / \mathrm{l}$ & 62 \\
II & $\begin{array}{l}\text { Albumin }>35 \mathrm{~g} / \mathrm{I} \\
\text { Not I or III }\end{array}$ & 45 \\
III & $\beta_{2}$ microglobulin $>5.5 \mathrm{mg} / \mathrm{l}$ & 29 \\
\hline
\end{tabular}

In addition to the laboratory tests, patients should have an annual skeletal survey and if the patient has known urinary free light chains, these should be quantified periodically. Thus far, early intervention before the development of symptomatic myeloma has shown no benefit in two randomised control trials. $^{715}$ Large centres treating myeloma are conducting clinical trials to determine whether newer agents such as thalidomide in combination with bisphosphonates can delay disease progression in asymptomatic myeloma.

\section{MANAGEMENT OF SYMPTOMATIC MYELOMA}

The purpose of this article is not to provide a practical guide to myeloma therapy but rather an overview of strategy. The management of symptomatic myeloma is complex and requires a range of specialist expertise from haematology, clinical oncology, nephrology, orthopaedics and palliative care. Without treatment the median survival of myeloma is 7 months and with chemotherapy this is increased to 3-4 years. Autologous stem cell transplantation can prolong overall survival by approximately 18 months in selected patients.

There have been several attempts to construct prognostic models since the Durie/Salmon staging system was devised in 1975. Currently the most useful system (international prognostic index, IPI) is based on the albumin and $\beta_{2}$ microglobulin levels (table 4). This is irrespective of the type of treatment selected. ${ }^{7}$

The treatment of myeloma is divided into supportive care with the goal of preventing or ameliorating complications of myeloma; these are most commonly renal, skeletal, infective and treatment related to bone marrow failure. The second aspect is specific treatment directed against the malignant plasma cells.

\section{SUPPORTIVE CARE}

- Pain control-Pain arising from the skeleton is the most common presenting complaint and will complicate $80 \%$ of patients during the course of their disease. The management requires both systemic treatment often with opioid analgesia and the use of local measures such as radiotherapy, orthopaedic, neurosurgical or interventional radiology-for example, vertebroplasty. Chemotherapy is also an important

Table 3 Risk stratification model to predict progression of monoclonal gammopathy of undetermined significance (MGUS) to myeloma or related disorders

\begin{tabular}{llcccc}
\hline & $\begin{array}{l}\text { Number } \\
\text { of patients }\end{array}$ & $\begin{array}{l}\text { Relative } \\
\text { risk }\end{array}$ & $\begin{array}{l}\text { Absolute risk } \\
\text { of } 20 \text { years (\%) } \\
\text { Risk group }\end{array}$ & $\begin{array}{l}\text { Absolute risk of progression } \\
\text { at 20 years accounting for } \\
\text { death as a competing risk (\%) }\end{array}$ \\
\hline $\begin{array}{l}\text { Low risk serum M-protein }<15 \mathrm{~g} / \mathrm{ll} \text {, IgG type, } \\
\text { normal free light chain ratio }(0.26-1.65)\end{array}$ & 449 & 1 & 5 & 2 \\
$\begin{array}{l}\text { Low-intermediate risk (any one risk factor } \\
\text { abnormal) }\end{array}$ & 420 & 5.4 & 21 & 10 \\
$\begin{array}{l}\text { High-intermediate risk (any two risk factors } \\
\text { abnormal) }\end{array}$ & 226 & 10.1 & 37 & 18 \\
High risk (all three risk factors abnormal) & 53 & 20.8 & 58 & 27 \\
\hline Reproduced with permission from Kyle and Rajkumar. & & &
\end{tabular}


part of pain control as it is aimed at the underlying pathological process.

- Hypercalcaemia-This occurs in up to $30 \%$ of patients typically in the presence of active disease-for example, at diagnosis or at relapse. Hypercalcaemia is managed with aggressive rehydration with saline and furosemide and commencement of bisphosphonates, or alternatively changing to a more potent bisphosphonate if already on treatment.

- Bisphosphonates-Bone pain, hypercalcaemia and pathological fractures are a major cause of morbidity and mortality in patients with myeloma. Long term bisphosphonates have been used increasingly to prevent these problems. Metaanalysis of trial data concluded that adding bisphosphonates reduces vertebral fractures and pain, but not mortality. Treatment with clodronate, pamidronate or zoledronate is therefore recommended for all patients with myeloma requiring chemotherapy, whether or not bone lesions are evident. There have been no published randomised trials comparing oral with intravenous bisphosphonates although currently this is being addressed in the UK MRC Multiple Myeloma IX trial which compares clodronate with zoledronic acid. At present the choice between oral and intravenous bisphosphonate is a matter of patient and physician preference. ${ }^{17} 18$

- Renal impairment-This occurs in up to $30 \%$ of patients at presentation and up to $50 \%$ of patients at some stage of the illness. The pathogenesis is multifactorial; light chain damage direct to the distal tubules, dehydration, sepsis, hypercalcaemia, hyperuricaemia and the use of nephrotoxic medications such as non-steroidal anti-inflammatory drugs. Patients are advised to keep well hydrated at all stages of the disease, drinking 3 litres of water per day, and avoiding potentially nephrotoxic agents. If renal failure does ensue, renal biopsy is desirable to guide management. The biopsy will identify those patients with acute tubular necrosis which may naturally resolve over time, amyloid and light chain deposition disease which does not respond to measures other than disease control, or cast nephropathy which may improve with rapid reduction of light chain concentration. Plasma exchange has been undertaken in cast nephropathy, however published trials have shown conflicting results. It is therefore appropriate that new patients are treated within a trial setting, such as the current UK MERIT trial (NCRN trial portfolio ISRCTN 37161699; http:// www.ncrn.org.uk/portfolio). In patients with established renal failure, dialysis should be offered where appropriate. ${ }^{7} 19$

- Anaemia-This is present in two thirds of patients at presentation and becomes more common with recurrent or progressive disease; it usually responds to control of the underlying myeloma. Traditionally, anaemia has been managed by transfusions of packed red cells although recombinant erythropoietin is being used increasingly. Studies have reported decreased transfusion requirements, increased haemoglobin concentrations and patient-assessed improved quality of life. Current recommendations suggest a trial of erythropoietin in patients on chemotherapy and those with symptomatic anaemia, with the aim of decreasing transfusion requirements. ${ }^{20} 21$

- Infections - The risk of infection is increased as a consequence of both disease and treatment. Contributory factors include the M-protein, immune paresis and disturbed B cell function. Steroids remain the mainstay of chemotherapy treatment but are associated with viral and fungal infections and the chemotherapy itself may produce variable degrees of neutropenia. A range of approaches are required, including $24 \mathrm{~h}$ access to specialist care and prompt intravenous broad spectrum antibiotic treatment for any febrile myeloma patient. Vaccination against Streptococcus pneumonia and Haemophilus influenzae, and prophylactic intravenous immunoglobulin in patients in plateau phase experiencing recurrent significant infections, may be helpful.

- Hyperviscosity-Symptomatic patients are managed with plasma exchange followed by prompt institution of chemotherapy.

- $A L$ amyloidosis-Approximately 15\% of patients with myeloma will develop AL amyloidosis with complications including cardiac failure, renal failure and neuropathy. There are recently published comprehensive UK guidelines for the evaluation and management of all AL amyloidosis patients including those with co-existent myeloma. ${ }^{22}$

\section{MYELOMA BIOLOGY AND SPECIFIC THERAPY}

In contrast to other haematological malignancies such as acute leukaemia and chronic myeloid leukaemia, until recently it has been difficult to identify the molecular events which underlie MGUS and myeloma. This was mainly due to the difficulty in performing cytogenetic analysis in these slowly proliferating tumours. Many of these difficulties have been resolved with the advent of or improvement in techniques such as: isolation and purification of plasma cells; interphase FISH cytogenetics; and the recognition of chromosomal translocations involving the immunoglobulin heavy chain switch region. From these studies it is emerging that cytogenetic abnormalities such as translocations are common in both MGUS (46\%), myeloma (55-73\%), and plasma cell leukaemia (85\%). Within plasma cell dyscrasias there appear to be two pathways involved in pathogenesis. Approximately half of cases have a karyotype that is nonhyperdiploid with a high incidence of both chromosome 13 loss, and an IgH switch region translocation involving one of five recurring partner chromosomal loci; 1lq13 (cyclin D1), 6p21 (cyclin D3), 4pl6 (fibroblast growth factor receptor 3 (FGFR3) and multiple myeloma SET domain (MMSET), 16q23 (c-maf) and 20q11 (mafB). The remaining tumours have a hyperdiploid karyotype with multiple chromosome trisomies. The translocations result in altered expression of oncogenes. There is an almost universal finding of increased expression of a cyclin D gene in MGUS and myeloma. Cyclin D overexpression may arise directly from the translocation involving the $\mathrm{IgH}$ switch region and either $11 \mathrm{q} 13$ or $6 \mathrm{p} 21$ partner loci; however, the mechanism of overexpression in hyperdiploid tumours or other translocations is unexplained. It is clear that certain karyotype aberrations, such as loss of chromosome 13 material and translocations involving $4 \mathrm{pl} 6$ and $16 \mathrm{q} 22$, predict a poor outcome and response to treatment. It is likely that in the future myeloma may be further classified on the basis of primary chromosomal translocation and cyclin expression, with more aggressive and/or experimental therapies being offered to those in the poorest prognostic group. ${ }^{23}$

\section{Useful websites}

$$
\begin{aligned}
& \text { Medical information } \\
& \text { - www.bcshguidelines.com } \\
& \text { - www.asheducationbook.org } \\
& \text { Patient information } \\
& \text { - www.Irf.org.uk } \\
& \text { - www.myeloma.org.uk } \\
& \text { - www.cancerbackup.org.uk }
\end{aligned}
$$


In current practice myeloma remains largely a disease of the elderly, with approximately 15\% of patients aged less than 60 years and fewer than $2 \%$ of cases arising in patients under 40 years old. This age distribution has implications for the types of treatment offered. Excluding a very few selected young patients aged less than 50 years for whom an allogeneic stem cell transplant may offer the prospect of cure, for the majority of patients current treatment is not curative and is aimed at prolonging good quality life. The optimal approach to achieving this is high dose therapy and autologous stem cell transplantation (HDT), an approach which is usually reserved for patients aged less than 65 years or those aged less than 70 with an excellent performance status. All other patients are treated with oral therapies. ${ }^{72}$

The treatment of patients eligible for HDT will consist of 46 months of cyclical combination chemotherapy via a central indwelling catheter. In the UK perhaps the most widely used regimen is a continuous 4 day intravenous infusion of vincristine and doxorubicin along with 4 day blocks of oral dexamethasone at a dose of $40 \mathrm{mg} /$ day. This regimen termed VAD is associated with a $60-70 \%$ response rate. Related infusional regimens using cyclophosphamide, vincristine, adriamycin and prednisolone (C-VAMP) are also widely used though there has been no randomised comparison with VAD. ${ }^{25} 26$ Two key points to note are: VAD is chosen in preference to oral alkylating agents such as melphalan as a pre-HDT chemotherapy regimen as it achieves rapid cytoreduction without prejudicing stem cell mobilisation. Many authorities consider that dexamethasone is the effective agent in these regimens and in many US centres high dose dexamethasone alone is used as initial chemotherapy. ${ }^{27}$ Since the introduction of thalidomide into myeloma chemotherapy regimens (described more fully below), US centres are increasingly using the combination of thalidomide and dexamethasone as induction therapy. Following initial chemotherapy patients will then undergo chemotherapy mobilised peripheral blood stem cell collection (PBSC) followed by high dose therapy using intravenous melphalan and PBSC rescue. The evidence base for HDT therapy is derived from four prospective randomised trials comparing conventional chemotherapy (CC) with HDT. A French multicentre study IFM90 randomised 200 patients and noted a median event-free survival for CC of 18 months and HDT 28 months, and overall survival for CC of 44 months and HDT 57 months. The other studies confirmed an improved outcome in their HDT arm though clearly the HDT approach is not curative and inevitably all patients will relapse. ${ }^{78} 29$

Patients ineligible for HDT are treated with oral chemotherapy. Early Medical Research Council trials in the 1970s established oral melphalan and prednisolone (MP) as an effective regimen. Subsequent meta-analysis showed that this combination is as effective as other regimens including intravenous chemotherapy. Consequently standard oral chemotherapy remains as cycles of oral MP administered until the patient achieves a maximum response plus a further 3 months. ${ }^{30} 31$

Inevitably all patients will relapse; if the duration of first plateau phase is greater than 6-12 months then re-treating with the same first line therapy may be effective. However, for many patients there is a need for new treatments. Two new agents are acquiring a role in the management of relapsed myeloma: thalidomide and bortezomib. There is greater experience with thalidomide. Thalidomide is an orally bioavailable glutamic acid derivative; among its biological activities it inhibits production of tumour necrosis factor $\alpha(\mathrm{TNF} \alpha)$ and angiogenic cytokines. On the basis of the known dependence of malignant plasma cells on bone marrow supporting cells and observed increased angiogenesis in marrow biopsies from myeloma cases, empirical therapy of myeloma using thalidomide

\section{Key references}

- Kyle RA, Rajkumar SV. Monoclonal gammopathy of undetermined significance. $\mathrm{Br} J$ Haematol 2006;134: 573-89.

- Smith A, Wisloff F, Samson D. Guidelines on the diagnosis and management of multiple myeloma 2005. Br J Haematol 2006; 132:410-51.

- Child JA, Morgan GJ, Davies FE, et al. High-dose chemotherapy with hematopoietic stem-cell rescue for multiple myeloma. N Engl J Med 2003;348:1875-83.

- Barlogie B, Desikan R, Eddlemon P, et al. Extended survival in advanced and refractory multiple myeloma after single-agent thalidomide: identification of prognostic factors in a phase 2 study of 169 patients. Blood 2001;98:492-4.

- Barlogie B, Shaughnessy J, Tricot G, et al. Treatment of multiple myeloma. Blood 2004;103:20-32.

was undertaken, and these initial studies showed that myeloma is responsive to thalidomide. ${ }^{32}$ Current practice is to use thalidomide for patients who relapse early after initial chemotherapy. Published studies of thalidomide as a single agent report a response rate of $30 \%$ or combined with dexamethasone a response rate of $60 \% .^{33}{ }^{34}$ Current relapse/refractory protocols use thalidomide combined with dexamethasone and cyclophosphamide. Thalidomide does have a significant side effect profile with frequent or severe problems being sedation, peripheral neuropathy, constipation and, when used in conjunction with dexamethasone, a high incidence of thromboembolic events. In view of the teratogenic effects of thalidomide, where required barrier forms of contraception are essential and care must be taken to ensure that the drug is only taken by the individual for whom it is prescribed. In view of the efficacy of thalidomide in relapsed/refractory myeloma, ongoing trials are addressing the question whether thalidomide should play a role in first line treatment of myeloma. Indeed, in the USA, outside clinical trials thalidomide and dexamethasone is now widely used as a first line therapy.

In view of the significant side effect profile of thalidomide, analogues of this agent have been developed. Lenalidomide is a third generation immunomodulatory drug which retains anti$\mathrm{TNF} \alpha$, immunomodulatory and anti-cancer activity. It does not cause sedation or constipation, and has a reduced incidence of neuropathy. The prothrombotic effect remains and there is a greater myelosuppressive effect than is seen with thalidomide. In clinical trials of relapsed refractory myeloma, a combination of lenalidomide and dexamethasone has a response rate of $38 \%$. Randomised trials of lenalidomide and dexamethasone compared to placebo and dexamethasone have shown a time to progression of 11.3 months versus 4.7 months, with a survival advantage in the lenalidomide arm. It is likely that lenalidomide, with an improved side effect profile compared to thalidomide, will soon be licensed in Europe as a second line therapy for myeloma. ${ }^{35}$

Bortezomib is an inhibitor of ubiquitin-mediated proteasome degradation. It is effective in relapsed myeloma and in a randomised study of 607 patients produced a better response rate $(45 \%)$ and median 1 year survival $(89 \%)$ than dexamethasone alone $\left(26 \%\right.$ and $72 \%$, respectively). ${ }^{36}{ }^{37}$

\section{SUMMARY}

Identifying a paraprotein is a frequent finding in medical practice, with MGUS the most common clonal plasma cell 
disorder in the general population. It is essential to distinguish MGUS from asymptomatic and symptomatic myeloma as the natural history is so varied. For patients with symptomatic myeloma the disorder remains incurable; there are, however, well-defined treatment protocols which have been shown to prolong good quality life. These include high dose therapy for patients under the age of 65 (age 70 if excellent performance status) and oral melphalan for the older patient. Supportive therapies including bisphosphonates and erythropoietin decrease morbidity and improve quality of life. There is a clear therapeutic benefit from thalidomide in myeloma and a current question under study is what role thalidomide may have in the future as part of first line therapy for symptomatic myeloma.

\section{MULTIPLE CHOICE QUESTIONS (TRUE (T)/FALSE (F); ANSWERS AFTER THE REFERENCES)}

(1) In individuals $>50$ years old, the incidence of paraproteinaemia is $3.2 \%$.

(2) In any individual with a circulating paraprotein, the finding of immunoglobulin light chains in the urine establishes the diagnosis of myeloma.

(3) In MGUS the presence of an abnormal serum free light chain ratio predicts an increased risk of progression to myeloma.

(4) High dose chemotherapy and autologous stem cell transplantation may cure a proportion of patients with asymptomatic myeloma.

(5) In patients with symptomatic myeloma the use of bisphosphonates is recommended only for those with radiological evidence of bone lytic lesions.

\section{ACKNOWLEDGEMENTS}

We are grateful to Dr Amin Rahemtulla for constructive comments on this article.

\section{Authors' affiliations}

Lucy Cook, Donald H C Macdonald, Department of Haematology, Imperial College, London, UK

Competing interests: None declared

\section{REFERENCES}

Kyle RA, Therneau TM, Rajkumar SV, et al. Prevalence of monoclonal gammopathy of undetermined significance. N Engl J Med 2006;354:1362-9

2 Landgren O, Gridley G, Turesson I, et al. Risk of monoclonal gammopathy of undetermined significance (MGUS) and subsequent multiple myeloma among African American and white veterans in the United States. Blood 2006; 107:904-6.

3 Kyle RA, Rajkumar SV. Monoclonal gammopathy of undetermined significance. Br J Haematol 2006; 134:573-89.

4 Oscier D, Fegan C, Hillmen P, et al. Guidelines on the diagnosis and management of chronic lymphocytic leukaemia. Br J Haematol 2004; 125:294-317.

5 Jaffe ES, World Health Organization. Pathology and genetics of tumours of haematopoietic and lymphoid tissues. Lyon and Oxford: IARC Press, Oxford University Press (distributor), 2001.

6 Merlini G, Stone MJ. Dangerous small B-cell clones. Blood 2006;108:2520-30.

7 Smith A, Wisloff F, Samson D. Guidelines on the diagnosis and management of multiple myeloma 2005. Br J Haematol 2006;132:410-51.

8 Lamboley V, Zabraniecki L, Sie P, et al. Myeloma and monoclonal gammopathy of uncertain significance associated with acquired von Willebrand's syndrome. Seven new cases with a literature review. Joint Bone Spine 2002;69:62-7.

9 Ropper AH, Gorson KC. Neuropathies associated with paraproteinemia. NEngl J Med 1998:338:1601-7.

10 Kyle RA, Therneau TM, Rajkumar SV, et al. A long-term study of prognosis in monoclonal gammopathy of undetermined significance. N Engl J Med 2002;346:564-9.
11 Blade J, Lopez-Guillermo A, Rozman C, et al. Malignant transformation and life expectancy in monoclonal gammopathy of undetermined significance. Br J Haematol 1992;81:391-4.

12 Cesana C, Klersy C, Barbarano L, et al. Prognostic factors for malignant transformation in monoclonal gammopathy of undetermined significance and smoldering multiple myeloma. J Clin Oncol 2002;20:1625-34.

13 Mead GP, Carr-Smith HD, Drayson MT, et al. Serum free light chains for monitoring multiple myeloma. Br J Haematol 2004; 126:348-54.

14 Rajkumar SV, Kyle RA, Therneau TM, et al. Serum free light chain ratio is an independent risk factor for progression in monoclonal gammopathy of undetermined significance. Blood 2005;106:812-17.

15 Hiorth M, Holmberg E, Rodjer S, et al. Impact of active and passive exclusions on the results of a clinical trial in multiple myeloma. The Myeloma Group of Western Sweden. Br J Haematol 1992;80:55-61.

16 Riccardi A, Mora O, Tinelli C, et al. Long-term survival of stage I multiple myeloma given chemotherapy just after diagnosis or at progression of the disease: a multicentre randomized study. Cooperative Group of Study and Treatment of Multiple Myeloma. Br J Cancer 2000;82:1254-60.

17 Diulbegovic B, Wheatley K, Ross J, et al. Bisphosphonates in multiple myeloma. Cochrane Database Syst Rev 2002:CD003188.

18 McCloskey EV, MacLennan IC, Drayson MT, et al. A randomized trial of the effect of clodronate on skeletal morbidity in multiple myeloma. MRC Working Party on Leukaemia in Adults. Br J Haematol 1998;100:317-25.

19 Knudsen LM, Hiorth M, Hippe E. Renal failure in multiple myeloma: reversibility and impact on the prognosis. Nordic Myeloma Study Group. Eur J Haematol 2000;65:175-81

20 Gabrilove JL, Cleeland CS, Livingston RB, et al. Clinical evaluation of onceweekly dosing of epoetin alfa in chemotherapy patients: improvements in hemoglobin and quality of life are similar to three-times-weekly dosing. J Clin Oncol 2001; 19:2875-82

21 Glaspy J, Bukowski R, Steinberg D, et al. Impact of therapy with epoetin alfa on clinical outcomes in patients with nonmyeloid malignancies during cancer chemotherapy in community oncology practice. Procrit Study Group. J Clin Oncol 1997;15:1218-34.

22 Guidelines Working Group of UK Myeloma Forum, British Committee for Standards in Haematology, British Society for Haematology, Guidelines on the diagnosis and management of $\mathrm{AL}$ amyloidosis. $\mathrm{Br} \mathrm{J}$ Haematol 2004:125:681-700.

23 Hideshima T, Bergsagel PL, Kuehl WM, et al. Advances in biology of multiple myeloma: clinical applications. Blood 2004;104:607-18

24 Soutar R, Lucraft H, Jackson G, et al. Guidelines on the diagnosis and management of solitary plasmacytoma of bone and solitary extramedullary plasmacytoma. Br J Haematol 2004;124:717-26.

25 Abrahamson GM, Bird JM, Newland AC, et al. A randomized study of VAD therapy with either concurrent or maintenance interferon in patients with newly diagnosed multiple myeloma. Br J Haematol 1996;94:659-64.

26 Raje N, Powles R, Kulkarni S, et al. A comparison of vincristine and doxorubicin infusional chemotherapy with methylprednisolone (VAMP) with the addition of weekly cyclophosphamide (C-VAMP) as induction treatment followed by autografting in previously untreated myeloma. Br J Haematol 1997;97:153-60.

27 Kumar S, Lacy MQ, Dispenzieri A, et al. Single agent dexamethasone for prestem cell transplant induction therapy for multiple myeloma. Bone Marrow Transplant 2004;34:485-90

28 Attal M, Harousseau JL, Stoppa AM, et al. A prospective, randomized trial of autologous bone marrow transplantation and chemotherapy in multiple myeloma. Intergroupe Francais du Myelome. N Engl J Med 1996;335:91-7.

29 Child JA, Morgan GJ, Davies FE, et al. High-dose chemotherapy with hematopoietic stem-cell rescue for multiple myeloma. N Engl J Med 2003;348:1875-83.

30 Combination chemotherapy versus melphalan plus prednisone as treatment for multiple myeloma: an overview of 6,633 patients from 27 randomized trials. Myeloma Trialists' Collaborative Group. J Clin Oncol 1998;16:3832-42.

31 Galton DA, Peto R. A progress report on the Medical Research Council's therapeutic trial in myelomatosis. Br J Haematol 1968;15:319-20.

32 Richardson PG, Mitsiades CS, Hideshima T, et al. Novel biological therapies for the treatment of multiple myeloma. Best Pract Res Clin Haematol 2005;18:619-34.

33 Barlogie B, Desikan R, Eddlemon P, et al. Extended survival in advanced and refractory multiple myeloma after single-agent thalidomide: identification of prognostic factors in a phase 2 study of 169 patients. Blood 2001;98:492-4.

34 Palumbo A, Giaccone L, Bertola A, et al. Low-dose thalidomide plus dexamethasone is an effective salvage therapy for advanced myeloma. Haematologica 2001;86:399-403.

35 Mazumder A, Jagannath S. Thalidomide and lenalidomide in multiple myeloma. Best Pract Res Clin Haematol 2006;19:769-80.

36 Richardson PG, Barlogie B, Berenson J, et al. A phase 2 study of bortezomib in relapsed, refractory myeloma. N Engl J Med 2003;348:2609-17.

37 Richardson PG, Schlossman R, Hideshima T, et al. New treatments for multiple myeloma. Oncology (Williston Park) 2005;19:1781-92; discussion 1792, 1795-87.

\section{ANSWERS}

(1) $\mathrm{T}$, (2) F, (3) T, (4) F, (5) F 